\title{
A IMPORTÂNCIA DA MOTIVAÇÃO E DO \\ TREINAMENTO NAS ORGANIZAÇÕES
}

\author{
THE IMPORTANCE OF MOTIVATION AND TRAINING IN \\ ORGANIZATIONS
}

Ana Paula Teodora Gomes ${ }^{1}$

\section{RESUMO}

Esta pesquisa tem o objetivo de descrever a influência da motivação e o impacto do treinamento nas organizações. É um trabalho de caráter bibliográfico, em que se busca a associação entre a motivação e os resultados de treinamentos aplicados nas organizações. A motivação poderá ser intrínseca ou extrínseca e possibilita verificar o quanto um profissional está disposto a investir na organização em que trabalha mediante o desempenho das tarefas executadas. Os treinamentos dentro das organizações são importantes para a melhoria da qualidade dos colaboradores; consequentemente, eles podem apresentar melhores resultados no desempenho de suas atividades. Os treinamentos bem planejados e executados, aliados à motivação e iniciativa (atitude) dos treinandos poderão gerar bons resultados, implicando no retorno esperado por parte das organizações que investiram na qualificação/treinamentos de seus colaboradores.

Palavras-chave: Motivação. Organização. Treinamento.

\section{ABSTRACT}

This research aims to describe the influence of motivation and the impact of training on organizations. It is a work of bibliographic character, in which the search between the motivation and the results of the training applied in the organizations is sought. The motivation may be intrinsic or extrinsic and makes it possible to verify how much a professional is willing to invest in the organization in which he works by performing the tasks performed. Training within organizations is important for improving the quality of employees; consequently, they can present better results in the performance of their activities. Well-designed and executed trainings, combined with the motivation and initiative (attitude) of the trainees, can generate good results, implying the expected return on the part of the organizations that invested in the qualification / training of their collaborators.

Keywords: Motivation. Organization. Training.

'Cat1 Tecnologia-Mineiros, Brasil. E-mail: anna_paulatg@hotmail.com 


\section{INTRODUÇÃO}

Existe dentro de diversas áreas um fator que está relacionado com as mudanças enfrentadas por pessoas e empresas: a globalização, que provocou uma maior competitividade no mercado principalmente desde o início do século XXI. Neste cenário, as organizações, para se manterem no mercado, necessitam de investimentos, incluindo a gestão de pessoas.

Segundo Depieri (2006), a área de gestão de pessoas tem assumido cada vez mais um papel estratégico nas organizações, tornando a mão de obra humana o diferencial diante da padronização dos produtos, dos preços e da qualidade no mercado competitivo. Quanto a esta questão, as empresas devem entender que há dois fatores importantes para o sucesso de qualquer equipe de colaboradores, que são a motivação e os treinamentos (capacitação dos trabalhadores). O treinamento é compreendido como

[...] uma ação direcionada para atividades atualmente desempenhadas, o desenvolvimento pode serentendido como ação orientada para o crescimento pessoal e profissional do funcionário e sem vínculo estreito com as atividades, presentes ou futura, demandas por determinada organização e a educação é uma ação voltada para atividades que serão desempenhadas em um futuro breve (MENESES;ZERBINI; ABBAD, 2010, p. 17).

Existem atualmente investimentos para propiciar o desenvolvimento de competências individuais, através de processo de aprendizagem, a fim de atingir os objetivos organizacionais. Os investimentos se justificam, segundo Azevedo (2006), devido à crença de que o maior diferencial competitivo no mercado de trabalho é força de trabalho qualificada. Busca-se cada vez mais valorizar os talentos individuais e o capital humano que são bens preciosos das organizações, por gerarem ideias, melhorarem processos e propiciarem resultados que atendem às expectativas e demandas dos clientes e, consequentemente, melhor rentabilidade para as empresas.

A aprendizagem possibilita aprimorar 0 desempenho individual dentro da organização, além de capacitar os indivíduos - através de novas tecnologias e conhecimentos - para possíveis novas funções. No Brasil, a Associação Brasileira de Treinamento e Desenvolvimento (ABTD, 2009) informa que o investimento anual médio com treinamentos vem crescendo em patamares superiores ao crescimento da economia, o que é uma demonstração da preocupação das organizações em se manterem atualizadas.

Em diversas áreas do conhecimento, como a Psicologia, a Sociologia, a Engenharia, a Educação, dentre outras, procura-se qualificar cada vez mais a mão de obra para o desenvolvimento e realização das atividades inerentes a cada organização. Nesse sentido, a presente pesquisa tem o objetivo principal de descrever a influência das variáveis motivacionais e o impacto dos treinamentos realizados nas organizações. Para isto, foram adotados os procedimentos metodológicos descritos a seguir.

\section{MATERIAL E MÉTODO}

Este estudo é caracterizado como sendo de 
caráter bibliográfico e exploratório, no qual buscaram-se esclarecimentos a respeito da influência das variáveis motivacionais e o impacto dos treinamentos nas organizações. Utilizou-se para isso, a pesquisa bibliográfica, realizada em livros, revistas e artigos científicos com os seguintes descritores: motivação, resultados, treinamentos, organização, trabalho, produção e planejamento.

O percurso realizado durante este estudo incluiu a busca, seleção e leitura de artigos científicos, assim como de capítulos de livros. Também, foram realizados fichamentos e resumos dos textos relacionadas ao tema, que possibilitaram o aprofundamento da temática, apresentado nos resultados e discussão.

\section{RESULTADOS E DISCUSSÃO}

Na antiguidade, a motivação era tida como uma força determinada e imposta por outras pessoas, como os pais, os professores e os chefes. Porém, na atualidade, acredita-se que ela poderá estar relacionada com os fatores pessoais e individuais e surge a partir de uma determinada necessidade. O termo motivação, no seu sentido etimológico vem da palavra latina movere, que indica mover, remetendo a uma ação dinâmica na vida dos indivíduos (BERGAMINI, 1997).

Para autores como Robbins (2002), Dubrin (2003), Gondim (2004), Chiavenato (2005), a motivação está relacionada diretamente à ação humana, tendo uma dimensão complexa. No que tange à organização, Chiavenato (2005, p.273) salienta que;

As empresas possuem, entre outros desafios, um grande, que é motivar seus colaboradores. A motivação significa o desejo de exercer altos níveis de esforçoem direção a determinados objetivos organizacionais, condicionado pela capacidade de também satisfazer objetos individuais.

Estudar a dinâmica motivacional possibilita entender o comportamento humano, prever e até buscar o controle de determinadas atitudes das pessoas. Para os psicólogos da linha behaviorista, elementos relacionados às condutas individuais estão ligados à motivação. Cada indivíduo tem sua motivação afetada e estimulada por inúmeros fatores ambientais. Assim, no estudo da motivação, é relevante que se leve em consideração cada variável do comportamento humano, para que se possa utilizar os estímulos mais apropriados para o desenvolvimento da motivação nos seus diferentes contextos, a exemplo das organizações.

Marques (2015) estabelece um diferencial entre o que ele nomeia de "motivação intrínseca" e "motivação extrínseca" salientando que a primeira considera a percepção/crenças positivas que a própria pessoa passa a ter em relação a si mesmo dentro do ambiente de trabalho; seria, então, uma motivação interna, inerente a cada sujeito; a segunda seria a valorização da pessoa por parte da organização, ou seja, elementos externos ao sujeito despertariam sua motivação. Estabelecendo uma divisão para a motivação Marques (2015) ressalta que o despertar da motivação pode ser diferente em cada pessoa, conceituando-a como

[...] a força que impulsiona o indivíduo a movimentar-se em direção a seus objetivos. O ser humano precisa se sentir motivado para fazer as atividades simples do cotidiano. É indispensável, portanto, estar atento ao que 
estimula essa sensação de motivação. Este sentimento de motivação e engajamento pode ser despertado de diversas formas, que podem ser divididas entre motivação intrínseca e extrínseca (MARQUES, 2015, p.el).

Ampliando as ideias sobre motivação Gondim (2004, p.146), compreende que se trata de um processo psicológico bastante complexo, por ser algo subjetivo e de difícil observação e que retrata de forma adequada cada ação dos indivíduos.

Já Robbins (2002, p.151) indica que a motivação é algo intenso que direciona as pessoas e procurarem atingir suas metas, ou em outras palavras, oesforço que elas fazem para se alcançar algo. Nessa linha de raciocínio, Chiavenato (2005, p.273), expõe que a motivação depende de uma direção, de força e intensidade do comportamento, de duração por um determinado período e persistência. Estes fatores por sua vez estão ligados a objetos, ao esforço e à necessidade que leva o sujeito a agir. Ainda lembra que "uma necessidade significa uma carência interna da pessoa e cria um estado de tensão no organismo. Daí o processo motivacional".

Portanto, a motivação intrínseca depende única e exclusivamente do próprio indivíduo, ou seja, é uma motivação que surge internamente, sem depender de reconhecimento ou incentivo de outras pessoas. Já a motivação extrínseca por sua vez, trata-se de estímulos que vêm de fora para dentro (externos), através de premiações, benefícios ou outras formas de recompensa por determinada tarefa atingida. Este tipo é utilizado por muitas empresas paramanter os empregados focados e engajados nos objetivos da organização. Apesar de serem tipos de motivação diferentes, são interligados e devem caminhar lado a lado, com a existência de um equilíbrio entre eles.

A motivação foi alvo de abordagem pela filosofia grega, porém no século XX começou a ser vista como uma abordagem psicológicagerencial (RÉGIS, 2000). Assim, tem-se procurado definir o comportamento humano através do estudo das variáveis dos indivíduos relacionados com os aspectos psicológicos.

A motivação, desta forma está ligada a fatores como a satisfação, o desejo, a energia, as recompensas intrínsecas e extrínsecas, o comprometimento, o envolvimento, o ajustamento no trabalho, o reforço, a necessidade, o desenho de cargo, as crenças, os valores, as metas, a expectativa e também a criatividade, a cultura, o afeto e o trabalho em equipes (AMBROSE; KULIK, 1999, apud GONDIM; SILVA, 2007).

De acordo com Vroom (1964), apud Lacerda e Abbad (2003), em sua teoria da expectância, que diz respeito a uma teoria cognitiva de processo, a motivação é vista dentro de um modelo multiplicativo, modelo este que pensa na tomada de decisão da pessoa considerando três variáveis: a valência, a instrumentalidade e a expectância.

A valência determina o quanto um indivíduo deseja ter uma recompensa ou realizar uma escolha em relação a um resultado particular. Também, pode ser vista como os valores positivos ou negativos de cada indivíduo em relação aos resultados desejados.

A instrumentalidade, por sua vez, se refere 
à estimativa de que um determinado desempenho seja um caminho adequado para se chegar a uma recompensa. Ela, porém, está ligada aos desejados desejos, crenças e à autoeficácia, pois se baseia no quanto a pessoa acredita ser possível alcançar o resultado que está sendo esperado.

Já a expectância tem como objetivo estimar o esforço que um indivíduo realiza para chegar a um resultado bem-sucedido, ou também a chance que este desempenho possa produzir no resultado desejado. A partir desta teoria, a motivação está intimamente ligada às escolhas do indivíduo e aos benefícios do programa de treinamento para o próprio treinando, relacionados a resultados futuros.

Quanto à associação entre motivação e aprendizagem Abbad, Pantoja e Pilati (2003) indicam que a motivação para o treinamento está relacionada com três níveis individuais de avaliação (que são as variáveis motivacionais). Elas se mostram como importantes fontes de previsão do sucesso individual nos treinamentos e são categorizados em níveis que são: reação, aprendizagem e impacto do treinamento no trabalho.

Também a motivação está ligada à vontade de aprender de cada indivíduo. De acordo com Lacerda e Abbad (2003) a motivação para aprender é determinada pelo interesse dos treinandos pelo conteúdo do programa de treinamento, bem como da persistência de cada um para se empenhar nas atividades orientadas, antes, durante e depois do treinamento.

Estes pesquisadores abordam ainda a motivação para transferir, que indica o grau de interesse do treinando em aplicar na prática, dentro de seu trabalho, os conteúdos aprendidos no treinamento. Em outras palavras o quanto o indivíduo está disposto a usar em sua rotina profissional o material visto no programa de treinamento.

\section{Treinamento nas Organizações}

O conceito de treinamento está relacionado com as muitas formas empregadas para se modificar o desempenho no trabalho, ou seja, uma tentativa de adquirir novos conhecimentos, habilidades e atitudes, além de uma maior compreensão das tarefas a serem executadas (COSTA, 2014). O treinamento se revela de suma importância para a qualidade dos resultados apresentados por uma equipe de trabalho de uma organização, pressupondo que o desempenho individual ou coletivo pode ser sempre melhorado através do treinamento e do desenvolvimento.

Além dos treinamentos convencionais, ligados à qualificação da mão de obra, surgiram também os treinamentos motivacionais, que tiveram início com a necessidade de se buscar uma conscientização para melhorar o comportamento dos indivíduos dentro das organizações (COSTA, 2014).

Conhecendo a necessidade de gerar programas que desenvolvam capacidades requeridas em futuras realidades, as tecnologias de treinamento foram desenvolvidas com a pretensão de identificar quais seriam estas necessidades futuras de capacitação (BORGES- ANDRADE, 1996). Em virtude das grandes mudanças dos processos de trabalho e das buscas por melhores desempenhos nas organizações, a 
tecnologia procurou responder a esta realidade no mundo do trabalho.

Já as estratégias do treinamento que buscam suprir as mudanças drásticas no perfil das empresas estão ligadas ao conceito de resultados, que devem ser medidos e estudados dentro das organizações. Borges-Andrade et al. (2006) argumentam que esta medida faz parte dos principais interesses em pesquisas que podem ser classificados em dois tipos: 1) examinar o efeito do treinamento em longo prazo sobre o desempenho dos treinados; 2) identificar fatores restritivos e facilitadores do uso das habilidades ensinadas e as condições necessárias para que o desempenho melhore.

Esta melhoria, no entanto, necessita de grandes investimentos financeiros, bancados geralmente pelas organizações que se preocupam com sua evolução e com a satisfação dos indivíduos em trabalhar nelas. Os treinamentos têm um papel notável no desenvolvimento e realização do trabalho realizado pelas pessoas dentro das organizações. Sabe-se que cada vez mais são necessários novos conhecimentos, habilidades e atitudes que propiciem novos produtos e serviços, com mais qualidade. Para atender a esta necessidade utilizam-se treinamentos como uma solução que vem crescendo, devido à busca por inovação e mudanças dentro dos processos e tarefas desempenhados nas organizações.

Neste sentido, o treinamento pode ser visto como uma oportunidade para melhorar o desempenho no cargo atual ou em cargos futuros do colaborador. No cenário atual, ele deixou de ser uma decisão pessoal, passando a ser uma necessidade de muitas organizações que possuem uma estratégia organizacional definida.

Ainda, é relevante pontuar a possibilidade de melhorias promovidas mediante o treinamento continuarem a acontecer de forma natural, uma vez que as pessoas aprendem através de observações e conversas com colegas de trabalho. Assim, os treinamentos poderão apresentar os desejados resultados pelas organizações que investiram em planejamento e na sua execução no ambiente de trabalho.

Por outro lado, a partir de Borges-Andrade (2002) verificam-se algumas dificuldade na aplicação de treinamentos dentro dos ambientes organizacionais, dificuldades essas que podem ser

desde a fase de preparação até no suporte necessário para a aplicação do conteúdo pretendido. As principais causas desta baixa eficácia dos treinamentos costumam estar associadas a fatores como a ausência de processos sistemáticos de levantamento de necessidades e de avaliação dos resultados, bem como ao uso de sistemas de treinamentos inadequados à realidade das organizações (BORGES-ANDRADE, 2002).

Buscando a reversão deste cenário, atualmente o treinamento é visto como fator de produção, que propicia uma vantagem competitiva, através de avaliações cada vez mais

constantes. Existe hoje em dia uma busca crescente por avaliação nas organizações que investem em treinamentos, que se dá devido às rápidas mudanças tecnológicas, econômicas e sociais ocorridas no mundo, no país, no ambiente de trabalho das organizações, exigindo o desenvolvimento de novas competências pessoais (BORGES-ANDRADE, 2002). 
Um treinamento depende de um bom planejamento. Abordando aspectos relacionados a esta variável, Vasconcellos (2013, p.el), pontua que, no planejamento do treinamento, deve-se considerar uma série de fatores, citados e explicados a seguir.

Identificação do cliente: fase inicial do planejamento do treinamento, quando se deve identificar de forma clara o perfil de cliente que se deseja atingir, ou seja, quem a organização precisa ou deseja atender. Deve haver uma preocupação com as necessidades dos clientes e com as soluções que a organização dispõe ou procura dispor para atendê-las.

Levantamento das necessidades: Deve-se também incluir no planejamento do treinamento as reais necessidades da organização. Não se devem procurar resultados imediatos, mas o treinamento possibilitará identificar os profissionais que podem ou não atender a estas necessidades. Nesta fase usam-se questionários para fazer avaliações de desempenho, discussões em grupo, reuniões entre os departamentos, entrevistas, pesquisas de ambiente, pesquisa de satisfação junto aos clientes, entre outras formas, para se conhecer as necessidades da empresa.

Diagnóstico do problema: neste ponto é possível diagnosticar os possíveis problemas encontrados em uma organização, como desvio de foco, problemas comportamentais, entre outros. Assim, poder-se-á determinar se há realmente a necessidade da realização de um programa de treinamento.

\section{Elaboração um-programa de}

treinamento: um bom programa de treinamento deve aliar identificação e interpretação das necessidades reais do treinamento, ou seja, o que levou ao fato de o treinamento ser realizado.

Público-alvo: nesta etapa deverá ser elaborada a relação de pessoas que serão atingidas com o treinamento. Também, devem ser divididos os conteúdos a serem aplicados dentro do programa, uma vez que o treinamento para o pessoal da parte técnica não será o mesmo da parte gerencial.

Objetivos: devem ser definidos claramente os objetivos do programa de treinamento, ou seja, o que se pretende alcançar com ele. Quando se tem dificuldades financeiras muitas empresas cortam verbas da área de treinamento, porque os objetivos e, consequentemente, seus resultados se mostram muito vagos. Por isso devem-se definir objetivos exequíveis e realistas, como estabelecer um desempenho final a ser alcançado, definir o tempo necessário para sua execução, etc.

Definição dos temas: assim, como ocorre com os objetivos, os temas a serem abordados devem ser claros, a fim de se atingir - da melhor forma possível - os resultados esperados.

Metodologia: é a forma pela qual o programa de treinamento será desenvolvido e executado. Alguns métodos existentes utilizam treinamentos a distância, pesquisas em internet, execução no próprio local de trabalho, conferências e palestras, estudos de caso, dinâmicas em grupo, aula expositiva dialogada, etc. Deve se escolher o método levando-se em consideração fatores como nível do treinando, necessidades a serem atendidas, tempodisponível para a realização do programa, recursos e materiais disponíveis, condições do ambiente, entre outros. Portanto, a metodologia deve atender 
à realidade da organização.

Os recursos a serem utilizados podem também variar como vídeos, quadros negros, cartazes, apostilas, computadores, slides-show, etc.

Tempo e custo: dois fatores imprescindíveis na elaboração do planejamento de treinamento são o tempo necessário para a realização e o custo que será dispendido para sua execução. Os elementos que influenciarão os custos são, por exemplo, os salários de instrutores e consultores externos, despesas com local do treinamento, materiais, etc. Devemos levar em consideração estes dois fatores antes de terminarmos a elaboração de um programa de treinamento para que não ocorram imprevistos que impossibilitem, por exemplo, a conclusão do treinamento.

\section{Execução do programa de treinamento:} aqui o planejamento de treinamento já está em fase de execução; esta parte inclui a convocação dos treinandos e a execução propriamente dita.

Convocação dos treinandos: para que o treinamento não se inicie com profissionais desmotivados, é necessário que - ao serem convocados - os participantes estejam cientes da importância da realização do treinamento e do aprendizado que eles terão. Deve-se também procurar convocar os trabalhadores através de maneiras mais criativas e chamativas e não mais através de cartazes, memorandos e outras maneiras formais.

Os instrutores: são responsáveis pela execução do treinamento, através da transmissão dos conteúdos teóricos e práticos do programa. Os instrutores devem ser verdadeiros agentes da mudança pretendida com o treinamento. A sua atuação poderá ser determinante para o sucesso ou o fracasso do treinamento. Deve possuir, dentre outras qualidades, personalidade, conhecimento do assunto, habilidade para lidar com confrontos de ideias, liderança, ética, entre outras. Deve saber, saber agir e saber ser ou seja, conhecer o que vai ensinar, procurar ensinar de modo a transmitir com a maior clareza possível os seus conhecimentos e ter uma postura pessoal ética, confiável, responsável e dinâmica.

O planejamento para os treinamentos éalgo relevante que deve ser levado a sério. As organizações entendem, cada vez mais, que existe a necessidade de realizá-los para se sobressaírem neste mundo marcado pela competitividade. Antes de se planejar um treinamento é necessário realizar um levantamento que procure identificar as possíveis necessidades, bem como avaliar se elas são reais ou não. Elas indicarão a necessidade ou não de se realizar o treinamento na organização. O setor de Recursos Humanos deve então assumir o papel de levantar necessidades e planejar o treinamento, com a realização da capacitação das pessoas, que é um dos pontos principais para o sucesso de qualquer tarefa.

Isto requer o Levantamento das Necessidades de Treinamento (LNT) que também proporcionará a melhoria da produtividade e da qualidade dos programas de treinamento $\mathrm{e}$ desenvolvimento humano. De acordo com Bezerra (2003) é necessário observar algumas condições importantes para a elaboração do LNT, entre elas, o planejamento que é um dos princípios fundamentais da Administração. O planejamento servirá para orientar o LNT e tem em vista a 
obtenção do máximo possível dos resultados a serem conseguidos.

Pode parecer simples, mas traçar os objetivos é algo sério que envolve a análise da demanda, consenso entre os envolvidos e demais aspectos que influenciarão positiva ou negativamente nos resultados. Devem contemplar: (i) os pessoais (dos colaboradores)e (ii) os organizacionais. Eles servem para realizar análises e confrontos, e consequentemente, possibilitam saber se estão existindo diferenças de ideias e valores no contexto organizacional. Além disto, os objetivos devem ser realistas, possíveis de serem realizados e claros ou poderão levar a realização de treinamentos falhos e vazios que não trarão benefícios para a organização (BEZERRA, 2003).

O acompanhamento do cumprimento dos objetivos é outro passo importante. Esta verificação facilita o processo de avaliação e o impacto que o treinamento trará, em outras palavras, o resultado do treinamento posto em prática de maneira diária na organização. A forma de verificação será a própria elaboração do banco de dados da organização. Este aspecto será avaliado pelo setor de Recursos Humanos, para saber se os objetivos foram ou não cumpridos dentro do que foi proposto. Este processo de avaliação também deve ser feito de forma clara, objetiva, realista e articulado ao que foi proposto.

Outro aspecto importante é o prazo de verificação para a avaliação do treinamento para se constatar ou não da sua eficácia. Em suma, é possível sugerir que um dos elementos e aspectos importantes para se alcançar um bom
LNT está na análise com precisão e de forma clara dos campos: objetivo e forma de verificação, que estão intimamente ligados e se forem considerados poderão diminuir a realização de treinamentos que não correspondam aos reais interesses da organização. A função de indicar treinamentos que sirvam para atender aos interesses da organização deve ser desempenhada pela parte gerencial em conjunto com a área de Recursos Humanos. O processo de Levantamento das Necessidades de Treinamento poderá ser realizado da maneira adequada quando seus elementos e etapas são considerados e seguidos.

\section{A Importância da Motivação e Treinamento nas Organizações}

A característica da clientela é um elemento dentro dos treinamentos que segundo Abbad (1999), é composta por variáveis motivacionais, cognitivas, demográficas e funcionais dos participantes dos treinamentos. As variáveis motivacionais envolvem a motivação para aprender e transferir valor, além do valor instrumental do treinamento. Com isto, observase que o treinamento e a motivação no contexto de organização estão interligados.

Assim, em qualquer treinamento é imprescindível que haja motivação. Neste sentido, Lacerda e Abbad (2003) definem motivação para treinamento como sendo o desempenho e as atitudes apresentadas pelos treinandos, ou seja, o quanto eles estão dispostos a aprender e transferir o aprendizado para os seus trabalhos cotidianos. Lacerda e Abbad (2003), esclarecendo sobre a motivação no treinamento consideraram três categorias: (a) Motivação para 
Aprender; (b) Motivação para Transferir; (c) e Valor Instrumental do Treinamento.

Motivação para aprender: está ligado ao interesse dos treinandos em querer aprender o que o programa estabelece como conteúdo do treinamento. Este interesse corresponde ao esforço, intensidade, direção e persistência que os treinandos demonstram nas atividades realizadas na aprendizagem antes, durante e também depois do treinamento.

Motivação para transferir: estabelece o grau de interesse que o treinando tem em aplicar na prática todo o conteúdo aprendido com o treinamento, ou seja, a disposição do indivíduo em usar na rotina cotidiana de seu trabalho, o conteúdo que foi aprendido no programa de treinamento.

Valor instrumental do treinamento: está ligado ao conceito de uma recompensa de várias naturezas (denominada de instrumentalidade), como sendo a utilidade que o treinamento possui na organização. Também corresponde à importância ou o valor que um indivíduo estabelece para cada recompensa (também chamada de valência) como importância do treinamento pelo qual ele passou.

Todos estes fatores ligados à motivação estabelecem o quanto terá êxito o desempenho do treinamento dentro da organização. E o desempenho que foi alcançado com o treinamento, segundo Abbad e Borges-Andrade (2004), engloba os fatores reflexivos: o quê? Como? Onde? Quando? Para quê? E com que padrões as tarefas são realizadas pelos indivíduos? O desempenho dentro do trabalho pode ser analisado como um resultado do conjunto das ações humanas, a fim de que se realizem as metas, que serão verificadas em termos de adequação, eficiência e eficácia.

A organização poderá verificar o impacto do treinamento, ao observar se os conhecimentos, habilidades e atitudes das pessoas resultarem em melhorias nos aspectos profissionais e pessoais. Abbad (1999) aponta o impacto do treinamento no trabalho como o terceiro nível de avaliação que envolve o efeito do treinamento no desempenho de cada indivíduo no contexto da organização. Este desempenho tem sido avaliado mediante a mensuração da transferência do treinamento, do desenvolvimento, da educação e da influência exercida no desempenho do treinado no decorrer da execução de suas tarefas.

$\mathrm{O}$ impacto propriamente dito se refere à influência que os eventos instrucionais terão sobre o desempenho dos participantes e representam o grau que eles aplicam em suas atividades profissionais, os conhecimentos, habilidades e atitudes, adquiridos no decorrer do treinamento. Borges-Andrade et al. (2006) apontam que o impacto é o resultado positivo da transferência de aprendizagem, onde há mudança de comportamentos e resultados no desempenho do sujeito que foi treinado, medidos através de indicadores de impacto que podem ser organizados em três categorias:

1. Comportamentos e resultados: são tidos como processos e operações desempenhadas pelos indivíduos treinados que buscam atingir diretamente o objetivo esperado para a melhoria destes processos e operações.

2. Complexidade da medida (profundidade ou amplitude): o impacto do treinamento no 
trabalhado pode ter duas medidas: uma em profundidade e outra em amplitude. Ambas expressam o conjunto de melhorias no desempenho em tarefas ligadas aos objetivos do treinamento. Porém o impacto em amplitude (largura) serve para medir os efeitos relacionados aos desempenhos gerais esperados pela organização. É uma especificação dos efeitos do treinamento de forma mais geral. Já o impacto em profundidade serve para distinguir os efeitos diretos dos efeitos específicos de um evento instrucional de outros considerados mais gerais. Desta forma, está mais ligado aos efeitos obtidos diretamente dos objetivos de ensino e dos conteúdos dos programas de treinamento.

\section{Nível a que se refere (indivíduo, grupos}

e organização): está relacionado aos momentos de medição, etapa do treinamento na qual foi feita a coleta dos dados e as variáveis que influenciam os resultados do impacto do treinamento. Abbad (1999) aponta que a avaliação do impacto do treinamento é feita através da observação do desempenho do indivíduo no trabalho, ou seja, da aplicação do que foi aprendido no ambiente de transferência.

O impacto no treinamento pode ser avaliado a partir de indicadores de resultados já utilizados pela organização ou então a partir de objetivos instrucionais ligados aos objetivos da empresa ou do setor do qual o treinando faz parte. Em síntese, o impacto do treinamento no trabalho envolve o desempenho e motivação, sendo preciso, além do saber fazer (habilidades), o querer fazer (motivação) a tarefa e ter condições no ambiente para sua realização. Quanto mais motivado o indivíduo estiver, melhor será a aplicação do aprendizado na prática, assim como o seu desempenho profissional e, consequentemente, o sucesso da empresa.

\section{CONSIDERAÇÕES FINAIS}

Esta pesquisa teve a intenção de descrever a influência das variáveis motivacionais e do impacto do treinamento nas organizações, baseada na sustentação teórica da relação entre motivação e treinamentos nas organizações, variáveis que podem gerar impacto na organização.

No processo de capacitação dos colaboradores é necessário que a Gestão de Pessoas realize um trabalho dinâmico, atualizado e competitivo, que possibilite modificações necessárias no ambiente de trabalho.

É necessário que os recursos investidos em treinamentos sejam utilizados de maneira correta, com programas que agreguem valores aos objetivos das organizações. Assim, é possível verificar uma relação entre motivação e os resultados de treinamentos, uma vez que a motivação aliada aos resultados dos treinamentos, pode impactar de forma objetiva e positiva os ambientes de trabalho e a dinâmica das organizações.

É possível sugerir que um treinamento adequado poderá despertar a motivação das pessoas nas organizações com sua melhoria e, consequentemente, se ter o aumento da produtividade dentro das empresas. Por outro lado, as práticas de treinamentos realizadas de maneira incorreta poderão gerar resultados inesperados, como o aumento de gastos para a organização, objetivos não atingidos, 
desmotivação dos gestores e dos próprios

colaboradores.

\section{REFERÊNCIAS BIBLIOGRÁFICAS}

ABBAD, G. S. Um modelo integrado de avaliação do impacto do treinamento no trabalho - IMPACT. Tese de doutorado, Instituto de Psicologia da Universidade de Brasília, Brasília, 1999.

ABBAD, G., P. R.; PANTOJA, M. J.. Avaliação de treinamento: análise da literatura e agenda de pesquisa. Revista de Administração - USP, 38(3), 181-91, 2003.

ABBAD, G. S.,; BORGES-ANDRADE, J. E.. Aprendizagem humana em organizações de trabalho. Im: ZANELLI, J.C. ; BORGESANDRADE, J. E.; BASTOS, A.V. B. (Orgs.). Psicologia organizações e trabalho no Brasil Porto Alegre: Artmed, 2004.

ABTD. O retrato do treinamento no Brasil. 2008/2009. Revista T\&D Inteligência Corporativa, 162, 2009.

AZEVEDO, L. P. S.. Comprometimento e bases de poder como preditores de impacto de treinamento no trabalho em contact center. Dissertação de mestrado, Instituto de Psicologia, Universidade Federal de Uberlândia, Uberlândia, 2006.

BERGAMINI, C. W. Motivação nas organizações. 4 ed. São Paulo: Atlas, 1997

\section{BEZERRA, L. H.. Levantamento das} Necessidades de Treinamentos. RH, Desenvolvimento, 2003. Disponível em: http://www.rh.com.br/Portal/Desenvolvimento/ Artigo/3683/levantamento-das-necessidadesde-treinamentos.html Acesso em 18/11/2014.

BORGES-ANDRADE, J. E. .

Desenvolvimento de medidas em avaliação de treinamento. Revista Estudos de Psicologia, 7 (especial), 31-43, 2002.

\section{CHIAVENATO, I.. Comportamento} Organizacional: a dinâmica do sucesso das organizações. 2 ed. Rio de Janeiro: Elsevier, 2005.
COSTA, A. B.. História e Evolução do Treinamento e Desenvolvimento. 2014 Disponível em:

<https://prezi.com/_d9hrobjuipc/historia-eevolucao-do-treinamento-e-desenvolvimento/ > Acesso em: 15/11/2014.

DEPIERI, M. A.. Impacto de educação corporativa: Educação continuada em processos educativos - FORFOR. Dissertação de mestrado, Faculdade de Economia, Administração, Contabilidade e Ciência de Informação e Documentação, Universidade de Brasília, 2006.

DUBRIN, A. J. Fundamentos do comportamento organizacional. São Paulo: Pioneira Thomson Learning, 2003.

FREITAS, I. A., BORGES-ANDRADE, J. E. ABBAD, G. S., PILATI, R. (2006). Medidas de impacto de TD\&E no trabalho e nas organizações. In: BORGES-ANDRADE, J. E; ABBAD, G.; MOURÃO, L. (org.). Treinamento, desenvolvimento e educação em organizações e trabalho: Fundamentos para a gestão de pessoas (pp. 489-504). Porto Alegre: ArtMed, 2006.

GONDIM, S.M.G.; SILVA, N. Motivação no trabalho. In: Zanelli, J. C.; Borges-Andrade, J. E.; Bastos, A.V. B (Orgs.). Psicologia, organizações e trabalho no Brasil. Porto Alegre: Artmed, 2004.

LACERDA, E. R. M.; ABBAD, G.. Impacto do treinamento no trabalho: investigando variáveis motivacionais e organizacionais como suas preditoras. Revista de Administração Contemporânea, 7(4), 77-96, 2003.

MARQUES, J. R.. Qual a diferença entre motivação intrínseca e extrínseca? 2015. Disponível em < http://www.jrmcoaching.com.br/blog/qual-adiferenca-entre-motivacao-intrinseca-eextrinseca/> Acesso em 11/07/2015

MENESES, P. P. M., ZERBINI, T.; ABBAD, G. . Manual de Treinamento Organizacional. Porto Alegre: Artmed, 2010.

RÉGIS, H. P.. A motivação dos professores do Centro Federal de Educação Tecnológica da Paraíba para participarem do programa de qualidade: um exame com base na teoria da expectância. Dissertação de Mestrado. Recife - 
Centro de Ciências Sociais Aplicadas -

Universidade Federal de Pernambuco, 2000.

ROBBINS, S. P.. Comportamento

Organizacional. 9 ed. São Paulo: Prentice Hall, 2002.

RODRIGUES, A. G. . A natureza da participação e suas implicações no Impacto de Treinamento no Trabalho. Dissertação de Mestrado. Brasília: Instituto de Psicologia Universidade de Brasília, 2000.

\section{VASCONCELlOS, J. E.. Como Planejar e} Executar um Treinamento. 2013. Disponível em:

<http://www.rhportal.com.br/artigos/rh.php?idc _cad=3hoanirfi $>$. Acesso em 16/11/2014.

\section{Como citar este artigo (Formato ABNT):}

GOMES, Ana Paula Teodora. A Importância da Motivação e do Treinamento nas Organizações. Educação, Psicologia e Interfaces, vol.1, n.1, p. 31-43, 2017. DOI: https://doi.org/10.37444/issn2594-5343.v1i1.15

Recebido: 10/02/2017.

Aceito: 02/03/2017. 